\title{
Three-Dimensional Distribution of the ISM in the Milky Way Galaxy: III. The Total Neutral Gas Disk
}

\author{
Hiroyuki NAKANISHI ${ }^{1,2}$ and Yoshiaki SOFUE ${ }^{3}$ \\ ${ }^{1}$ Graduate Schools of Science and Engineering, Kagoshima university, 1-21-35 Korimoto \\ Kagoshima \\ ${ }^{2}$ Institute of Space and Astronautical Science, Japan Aerospace Exploration Agency, 3-1-1 \\ Yoshinodai, Chuo-ku, Sagamihara, Kanagawa 252-5210, Japan \\ ${ }^{3}$ Institute of Astronomy, The University of Tokyo, 2-21-1 Osawa, Mitaka,Tokyo 181-0015 \\ *E-mail: hnakanis@sci.kagoshima-u.ac.jp
}

Received 2015 August 17; Accepted 2015 October 6

\begin{abstract}
We present newly obtained three-dimensional gaseous maps of the Milky Way Galaxy; $\mathrm{HI}, \mathrm{H}_{2}$ and total-gas $\left(\mathrm{HI}\right.$ plus $\left.\mathrm{H}_{2}\right)$ maps, which were derived from the $\mathrm{HI}$ and ${ }^{12} \mathrm{CO}(J=1-0)$ survey data and rotation curves based on the kinematic distance. The $\mathrm{HI}$ and $\mathrm{H}_{2}$ face-on maps show that the $\mathrm{HI}$ disk is extended to the radius of $15-20 \mathrm{kpc}$ and its outskirt is asymmetric to the Galactic center, while most of the $\mathrm{H}_{2}$ gas is distributed inside the solar circle. The total gas mass within radius $30 \mathrm{kpc}$ amounts to $8.0 \times 10^{9} \mathrm{M}_{\odot}, 89 \%$ and $11 \%$ of which are $\mathrm{HI}$ and $\mathrm{H}_{2}$, respectively. The vertical slices show that the outer $\mathrm{HI}$ disk is strongly warped and the inner $\mathrm{HI}$ and $\mathrm{H}_{2}$ disks are corrugated. The total gas map is advantageous to trace spiral structure from the inner to outer disk. Spiral structures such as the Norma-Cygnus, the Perseus, the Sagittarius-Carina, the Scutum-Crux, and the Orion arms are more clearly traced in the total gas map than ever. All the spiral arms are well explained with logarithmic spiral arms with pitch angle of $11^{\circ}-15^{\circ}$. The molecular fraction to the total gas is high near the Galactic center and decreases with the Galactocentric distance. The molecular fraction also locally enhanced at the spiral arms compared with the inter-arm regions.
\end{abstract}

Key words: Galaxy: disk — Galaxy: kinematics and dynamics — Galaxy: structure — ISM: kinematics and dynamics — radio lines: ISM

\section{INTRODUCTION}

The interstellar medium (ISM) is one of the major components of the Galaxy and consists of coronal gas, intercloud gas, diffuse clouds, dark clouds, Bok globules, molecular clouds, and H II regions (Myers 1978). The neutral hydrogen H I radiates 1420 $\mathrm{MHz}$ line and traces intercloud gas, diffuse clouds, and dark clouds. Though dominant component of molecular clouds is $\mathrm{H}_{2}$ gas, it does not have emission line in the radio wavelength range. Therefore, in general, carbon monoxide molecule $\mathrm{CO}$ line is used as a tracer of $\mathrm{H}_{2}$ gas and $\mathrm{H}_{2}$ gas density is calculated with a conversion factor $X_{\mathrm{CO}}$.

All sky HI and CO line survey data in radio wavelength are great tools to reveal the Galactic global structure, because (1) radio signal suffers relatively low extinction due to its long wavelength, (2) neutral hydrogen and molecular gases observed in the radio lines are distributed in the whole Galaxy, and (3) line data provide us with information about distances using a kinematic distance method.

There have been a lot of efforts to conduct $\mathrm{HI}$ and $\mathrm{CO}$ line surveys for the Galaxy. Muller \& Westerhout (1957) carried out Kootwijk HI survey with 7.5-m telescope, with which Oort et al. (1958) constructed the HI distribution map and found spiral structures such as the Sagittarius, the Orion (Local)), and the Perseus arms. Succeeding HI survey works found 
more spiral structures such as the Cygnus (Outer) arm by analyzing longitude-velocity (LV) diagram (Kerr 1969; Weaver 1970; Verschuur 1973). The latest HI survey conducted with Australia Telescope Compact Array showed that there exist additional spiral structure at the end of the HI disk in the fourth quadrant (McClure-Griffiths et al. 2004).

Dame et al. (1987) and Bronfman et al. (1988) carried out ${ }^{12} \mathrm{CO}(J=1-0)$ line survey for the Galactic plane with 1.2$\mathrm{m}$ telescope, with which the Sagittarius and Carina arms were clearly traced by locating giant molecular clouds in the Galactic plane (Cohen et al. 1985; Dame et al. 1986; Myers et al. 1986; Grabelsky et al. 1987). Sanders et al. (1986) carried out CO survey for the first quadrant of the Galaxy with 14-m telescope and traced the Sagittarius and Perseus arms. Using the same CO survey data, Clemens, Sanders, \& Scoville (1988) derived a face-on map of the molecular cloud distribution in the first quadrant. The latest study identified new distant molecular spiral arm in the first quadrant, which is considered to be the continuation of the ScutumCrux arm (Dame \& Thaddeus 2011).

These spiral arms are identified with those in many other tracers such as H II regions (Georgelin and Georgelin 1976; Downes et al. 1980), thermal electrons (Taylor \& Cordes 1993), dust emission (Drimmel and Spergel 2001), ${ }^{26} \mathrm{Al}$ (Chen et al. 1996), synchrotron emission (Beuermann et al. 1985), and methanol masers (Caswell et al. 2011) besides the HI and CO studies. Vallée (2005) compiled studies of measurements of spiral arms published from 1980 to early 2005 for the metaanalysis of the Galactic spiral structure and presented spiral arms. Most studies have identified four spiral arms, whose shapes are concluded as logarithmic spiral arms with pitch angle of $13 .{ }^{\circ} 1 \pm 0.6$ (Vallée 2015).

In Paper I (Nakanishi \& Sofue 2003) and Paper II (Nakanishi \& Sofue 2006), we derived $\mathrm{HI}$ and $\mathrm{H}_{2}$ gas maps using the latest HI (Hartmann \& Burton 1997; Kerr et al. 1986; Burton \& Liszt 1983) and CO survey data (Dame, Hartmann, \& Thaddeus 2001), and rotation curves (Clemens 1985; Dehnen and Binney 1998), based on the kinematic distance method. In these papers, we showed individual three dimensional maps of $\mathrm{HI}$ and $\mathrm{H}_{2}$ disks. The $\mathrm{HI}$ gas is vastly distributed in the Galactic disk and it is extended further beyond the edge of the stellar disk. The surface density of the HI gas is larger in the outer Galaxy than in the inner Galaxy. On the other hand, the most of the $\mathrm{H}_{2}$ gas is distributed within the stellar disk. Therefore, the HI data is advantageous to investigate the structure of the outer Galaxy, while CO data is suitable to examine the inner Galaxy.

In this paper we present total gas maps as well as $\mathrm{HI}$ and $\mathrm{H}_{2}$ as the third part of this series. The total gas map is suitable for investigating the entire structure of the Galaxy from the inner to outer part. At the same time, we revise the HI map using the newly released LAB survey data (Kalberla et al. 2005) after Paper I and it has been found that the data analysis method should be improved through the work of Paper II.

We choose a Cartesian coordinate whose $\mathrm{x}$-axis coincides with the line crossing the Sun and the Galactic center, and whose origin coincides with the Galactic center. The $z$-axis is chosen to be parallel to the rotational axis. We define that the Sun is located at $(-8 \mathrm{kpc}, 0,0)$. We also use cylindrical coordinates $(R, \theta, z)$ so that the angle $\theta=180^{\circ}$ coincides with the direction toward the Sun and the angle $\theta=90^{\circ}$ was parallel to $l=90^{\circ}$. The Galactic constants $R_{0}=8.0 \mathrm{kpc}$ (the Galactocentric distance of the Sun) and $V_{0}=217 \mathrm{~km} \mathrm{~s}^{-1}$ (the solar rotational velocity) are adopted (Dehnen and Binney 1998).

\section{Data}

HI survey data were taken from the LAB (Leiden-ArgentineBonn) survey (Kalberla et al. 2005), which consists of two survey data sets; Leiden/Dwingeloo Survey conducted with Leiden 25-m telescope whose half-power-beam-width (HPBW) was 0.6 covering the northern sky of $\delta \geq-30^{\circ}$ (Hartmann \& Burton 1997) and Instituto Argentino de Radioastronomia Survey conducted with $30-\mathrm{m}$ telescope whose HPBW was 0.5 covering the southern sky of $\delta \leq-25^{\circ}$ (Bajaja et al. 2005). The map grid spacing was 0.5 which corresponded to the linear scale of 17 $\mathrm{pc}$ at the heliocentric distance of $1 \mathrm{kpc}$, the LSR (local standard of rest) velocity coverage ranged from $-450 \mathrm{~km} \mathrm{~s}^{-1}$ to +400 $\mathrm{km} \mathrm{s}^{-1}$ at a resolution of $1.3 \mathrm{~km} \mathrm{~s}^{-1}$, and the rms noise in the brightness temperature was $0.07-0.09 \mathrm{~K}$. Residual systematic errors were reported as below $0.02-0.04 \mathrm{~K}$. In Paper I, we used only spectra within the latitude range of $|\delta| \leq 10^{\circ}$ because the data for the southern hemisphere covered only $|\delta| \leq 10^{\circ}$. However, we used spectra in the latitude range of $|\delta| \leq 30^{\circ}$, which covers more than $99 \%$ of the entire gaseous disk if we assume it is a cylinder with a radius of $20 \mathrm{kpc}$ and thickness of $2 \mathrm{kpc}$,

$\mathrm{CO}$ survey data, which are used to derive $\mathrm{H}_{2}$ gas distribution, were adopted from Dame, Hartmann, \& Thaddeus (2001), who compiled CO survey data from Grabelsky et al. (1987), Bronfman et al. (1989), May et al. (1993), Bitran et al. (1997) as described in Paper II. The data were obtained with 1.2-m telescopes in the USA and Chile. The HPBW was 8!.4-8.'8, which corresponds to $2.4-2.5 \mathrm{pc}$ at the heliocentric distance of $1 \mathrm{kpc}$. The map grid spacing was either $1 / 8^{\circ}$ or $1 / 4^{\circ}$ depending on a survey area. The LSR velocity coverage was $332 \mathrm{~km} \mathrm{~s}^{-1}$ and the velocity resolution was $1.3 \mathrm{~km} \mathrm{~s}^{-1}$. The rms noise in the brightness temperature was $0.12-0.43 \mathrm{~K}$. The Galactic latitude range is $|b| \leq 1 .^{\circ} 5$. Since most of the molecular gas is distributed inside the solar circle and the FWHM (full width of half maximum) of the molecular disk is typically $100 \mathrm{pc}$, most of the CO gas is covered with spectra in this range.

The rotation curve data are also the same as those we used 
in Papers I and II. The outer rotation curve $\left(R>R_{0}\right)$ was taken from Dehnen and Binney (1998), who presented model rotation curves fitted to the observation data available. The outer rotation curve we adopted is the best fitted model among them. The Galactic constant $R_{0}=8.0 \mathrm{kpc}$ and $V_{0}=217 \mathrm{~km} \mathrm{~s}^{-1}$ are adopted in this model. The inner rotation curve $\left(R<R_{0}\right)$ was taken from Clemens (1985), which was derived from the terminal velocity traced with the Massachusetts-Stoney Brook Galactic equator CO survey data. Though the original rotation curve was fitted with a polynomial function adopting Galactic constants of $\left(R_{0}, V_{0}\right)=\left(8.5 \mathrm{kpc}, 220 \mathrm{~km} \mathrm{~s}^{-1}\right)$ and $(10 \mathrm{kpc}, 250$ $\mathrm{km} \mathrm{s}^{-1}$ ), we modified the constants of the polynomial function for the case of $\left(R_{0}, V_{0}\right)=\left(8.0 \mathrm{kpc}, 217 \mathrm{~km} \mathrm{~s}^{-1}\right)$ in order to connect smoothly to the outer rotation as described in Paper I.

\section{Derivations of $\mathrm{HI}, \mathrm{H}_{2}$, and Total Gas Maps}

\subsection{Kinematic Distance}

Assuming that the gases circularly rotate around the Galactic center without non-circular motion and that the rotational velocity is constant against the height from the Galactic plane, the heliocentric distance $r$ of $\mathrm{HI}$ and $\mathrm{CO}$ gases were calculated based on the kinematic distance as follows.

The LSR velocity $V_{\mathrm{LSR}}$ of gas component observed in the direction $(l, b)$ rotating at the Galactocentric distance $R$ is given by the following equation,

$V_{\mathrm{LSR}}=\left[\frac{R_{0}}{R} V(R)-V_{0}\right] \sin l \cos b$

The heliocentric distance $r$ of the gas component can be given by solving the following relation of $r, R$ and $R_{0}$

$R^{2}=r^{2}+R_{0}^{2}-2 r R_{0} \cos l$.

In the case of the outer Galaxy $\left(R>R_{0}\right)$, the heliocentric distance is uniquely determined to be $r=R_{0} \cos l+$ $\sqrt{R^{2}-R_{0}^{2} \sin ^{2} l}$, while it has two solutions $r=R_{0} \cos l \pm$ $\sqrt{R^{2}-R_{0}^{2} \sin ^{2} l}$ for a given LSR velocity in the case of the inner Galaxy. However, there is no ambiguity at the tangential point $R=R_{0} \sin l$, where the LSR velocity reaches the maximum, which is refereed as terminal velocity $V_{\mathrm{t}}$, and the heliocentric distance is given by $r=R_{0} \cos l$.

\section{2 $\mathrm{HI}$ and $\mathrm{H}_{2}$ distributions in the outer Galaxy}

In the case of the outer Galaxy, the heliocentric distance can be uniquely determined as explained in the previous subsection. As described in Papers I and II, volume number densities $n\left[\mathrm{~cm}^{-1}\right]$ of $\mathrm{HI}$ and $\mathrm{H}_{2}$ gases at the heliocentric distance $r$ in the direction $(l, b)$ are calculated using the following equation,

$n=X T_{\mathrm{b}} \frac{\Delta V_{\mathrm{r}}}{\Delta r}$

where $X$ is the conversion factor, for which we adopt $X=$
$1.82 \times 10^{18}\left(\mathrm{H} \mathrm{cm}^{-2} \mathrm{~K}^{-1} \mathrm{~km}^{-1} \mathrm{~s}\right)$ for $\mathrm{HI}$ and $X=1.8 \times 10^{20}$ $\left(\mathrm{H}_{2} \mathrm{~cm}^{-2} \mathrm{~K}^{-1} \mathrm{~km}^{-1} \mathrm{~s}\right.$ ) for $\mathrm{H}_{2}$ gase (Dame, Hartmann, \& Thaddeus 2001), respectively. As described in Paper II, it is known that the conversion factor is likely to increase with Galactocentric distance (Arimoto, Sofue, \& Tsujimoto 1996). Therefore, the column density estimated in this paper might be overestimated around the Galactic center and underestimated for the outer Galaxy. $\mathrm{H}_{2}$ map obtained by adopting the conversion factor $X$ taken from Arimoto, Sofue, \& Tsujimoto (1996) is shown in Paper II.

\subsection{HI distribution in the inner Galaxy}

In the case of the inner Galaxy, there is near-far ambiguity that there are two points $\left(r_{1}, r_{2}\right)$ giving the same LSR velocity $V_{\mathrm{LSR}}$ in a single line-of-sight as explained in section 3.1. In order to solve this problem, we introduced a model of vertical density distribution at $r_{i}$ (Spitzer 1942),

$n_{i}(z)=n_{0 i} \operatorname{sech}^{2}\left(\ln (1+\sqrt{2}) \frac{z-z_{0 i}}{z_{1 / 2}}\right)$,

where $z_{0 i}$ is the height of a midplane from the plane of $b=0^{\circ}$, $n_{0 i}$ is volume density at the midplane, and $z_{1 / 2}$ is half the full width of half maximum (FWHM) of the vertical gas distribution.

Adopting this model, the observed column density $N(b)$ is given as a function of Galactic latitude $b$ with the above model as follows,

$N(b) \cos b=n_{1}\left(r_{1} \tan b\right) \Delta r_{1}+n_{2}\left(r_{2} \tan b\right) \Delta r_{2}$.

where the subscriptions 1 and 2 denote the near and far points, respectively.

Since the thickness of the gas layer $z_{1 / 2}$ primarily depends on the Galactocentric distance, for a given LSR velocity, unknown parameters are $n_{01}, n_{02}, z_{01}$, and $z_{02}$, which we can estimate by fitting the model function to the data. A searching range of $z_{01}$ and $z_{02}$ is restricted to $|z|<250 \mathrm{pc}$ as described in Paper II. To eliminate ill fitting results giving artificial structures, we smoothed the obtained distance-density curve using a spline function and clipping largely deviating points.

\subsection{Vertical profile of $\mathrm{HI}$ and $\mathrm{H}_{2}$ gases at tangential point}

Before applying the fitting method described in the previous subsection, we measured the thickness of the gas disk layer by analyzing the vertical ( $z$-direction) profile of $\mathrm{HI}$ and $\mathrm{H}_{2}$ gases at the tangential point, where the kinematic distance is uniquely determined for a given LSR velocity. Since the HI gas having an LSR velocity of the range $\left|V_{\mathrm{t}}\right|-\sigma \leq\left|V_{\mathrm{LSR}}\right| \leq\left|V_{\mathrm{t}}\right|$ contributes to a higher velocity component than the terminal velocity due the velocity dispersion $\sigma$, HI volume density was calculated by integrating the emission within the velocity range of 
$\left|V_{\mathrm{t}}\right|-\sigma \leq\left|V_{\mathrm{LSR}}\right| \leq \infty$ adopting $\sigma=5 \mathrm{~km} \mathrm{~s}^{-1}$ (Clemens 1985). Details are described in Papers I and II.

In the case of the outer Galaxy, the thickness was measured for all the points where vertical HI profile obtained.

The thickness $z_{1 / 2}$ was measured by fitting the model function to the observed vertical distributions. The measured FWHM $\left(2 z_{1 / 2}\right)$ of each gas layer is presented in section 4.4.

\subsection{Regridding the obtained $\mathrm{HI}$ and $\mathrm{H}_{2}$ distributions}

As described above, we obtained $\mathrm{HI}$ and $\mathrm{H}_{2}$ density cubes in the form of $n_{\mathrm{HI}}(l, b, r)$ and $n_{\mathrm{H}_{2}}(l, b, r)$, from the line spectra cube $T_{\mathrm{b}}\left(l, b, V_{\mathrm{LSR}}\right)$. Finally, these data cubes in the $(l, b, r)$ coordinates were transformed into $(x, y, z)$ coordinates explained in the section 1 .

For the outer Galaxy, the data were regridded by interpolating the nearest points. For the inner Galaxy, first the parameter sets of $z_{0}$ (midplane height) and $n_{0}$ (midplane density) were regridded by averaging ones surrounding a certain point with a weight function exponentially decreasing with FWHM of 0.48 $\mathrm{kpc}$ as described in Paper II.

The foreground $\mathrm{HI}$ emission surrounding the Sun having LSR velocity of $V_{\mathrm{LSR}} \sim 0 \mathrm{~km} \mathrm{~s}^{-1}$ can contribute to density distribution around the solar circle $R=R_{0}$ where the gas giving LSR velocity of $0 \mathrm{~km} \mathrm{~s}^{-1}$. To avoid this artificial effect, the data were regridded in the same way as the inner Galaxy for the region of $\left|V_{\mathrm{LSR}}\right|$ being less than $3 \sigma$, instead of simple interpolation used for the outer Galaxy.

\subsection{Revision of HI map}

In this paper, HI distribution map is revised. The following points were revised: (1) searching range of $z_{0}$ is restricted to $-250-+250 \mathrm{pc}$ the same as Paper II, in calculating HI distribution in the inner Galaxy, (2) the parameter sets $\left(n_{0}, z_{0}\right)$ obtained for the discrete points in the inner Galaxy were averaged with a Gaussian function whose FWHM was $0.48 \mathrm{kpc}$ in order to regrid HI distribution data, and (3) flare component of the outer HI disk is not subtracted. Due to (1) and (2), artificial structures seen in high-altitude of the inner Galaxy were able to be suppressed and the averaged surface density is not affected. Due to (3), the estimation of HI mass changed by factor of 2 . In Paper I, the most of the high-altitude emission was considered to be contributed from the local gas and this component was subtracted assuming that the local gas could be expressed by a linear function of the Galactic latitude $b$. Hence, the HI density at the midplane was also underestimated in this process. $\mathrm{H}_{2}$ distribution map is taken from the Paper II without any revision.

\section{New HI, $\mathrm{H}_{2}$, and Total Gas Maps}

\subsection{Face-on Maps}

Figure 1 shows contour maps of resulting $\mathrm{HI}$ and $\mathrm{H}_{2}$ column densities in the face-on view. The data range $|\theta|<30^{\circ}$ (i.e. behind the Galactic Center) was masked because data points are too sparse to make a map as described in Paper II. The global features such as spiral arms are consistent with previous works (Nakanishi \& Sofue 2003; Nakanishi \& Sofue 2006).

The $\mathrm{H}_{2}$ gas is concentrated in the inner region $\left(\lesssim R_{0} \mathrm{kpc}\right)$ and is hardly detectable in the outer region. On the other hand, the HI gas is distributed from the center to the outer region $(\gtrsim$ $R_{0} \mathrm{kpc}$ ). The lowest contour of $1 \mathrm{M}_{\odot} \mathrm{pc}^{-2}$, which is often referred as a radius of gaseous disk (Broeils \& van Woerden 1994), shows that the radius is $15-20 \mathrm{kpc}$ and that the outskirt is not axissymmetric. The HI disk swells in the direction of $\theta=-45^{\circ}$ if we assume a pure circular rotation. It is also shown that the disk is unnaturally asymmetry about $l=180^{\circ}$. It is likely that the outermost disk does not circularly rotate with the outer disk.

Next, we show comparisons of $\mathrm{HI}$ and $\mathrm{H}_{2}$ in figure 2. The top panel of figure 2 is a false color image showing $\mathrm{HI}$ and $\mathrm{H}_{2}$ in red and green, respectively. This figure shows obviously $\mathrm{HI}$ and $\mathrm{H}_{2}$ gases are dominant in the outer and inner parts, respectively. The gas phase transition from $\mathrm{HI}$ to $\mathrm{H}_{2}$ occurs within a narrow range of radius around $R_{0}$, which is referred as molecular front (Sofue, Honma, \& Arimoto 1995; Honma, Sofue, \& Arimoto 1995).

The bottom panel of figure 2 is a total ( $\mathrm{HI}$ plus $\mathrm{H}_{2}$ ) gas map, which is presented for the first time by obtaining both $\mathrm{HI}$ and $\mathrm{H}_{2}$ maps. Figure 3 shows a contour map of the total gas map shown in figure 2. These maps are advantageous to trace global structure such as spiral arms from the inner to the outer parts, since $\mathrm{H}_{2}$ and $\mathrm{HI}$ components preferentially traces the inner and outer arms, respectively. Spiral arms traced here are described in the section 4.5.

\subsection{Radial distribution}

Left panel of figure 4 shows radial distributions of the $\mathrm{HI}, \mathrm{H}_{2}$, and the total ( $\mathrm{HI}$ plus $\left.\mathrm{H}_{2}\right)$ gases, in filled circles, open circles, and solid line, respectively. This figure shows that the molecular gas is dominant in the central region and $\mathrm{HI}$ gas is dominant in the outer region. The total gas density attains its maximum at the center and second peak around $R=10 \mathrm{kpc}$. Beyond $R=10$ $\mathrm{kpc}$, it decreases with the Galactocentric distance and becomes lower than $1 M_{\odot} \mathrm{pc}^{-2}$ around $R=17 \mathrm{kpc}$. The dashed line is exponential function expressed with $\Sigma(R)=\Sigma_{0} e^{-\left(R-R_{0}\right) / R_{h}}$, where $\Sigma_{0}=30 \mathrm{M}_{\odot} \mathrm{pc}^{-2}$ and $R_{h}=3.75 \mathrm{kpc}$, adopted from Kalberla \& Kerp (2009).

Right panel of figure 4 shows the accumulated gas mass as a function of the Galactocentric distance. $\mathrm{HI}$ and $\mathrm{H}_{2}$ masses 

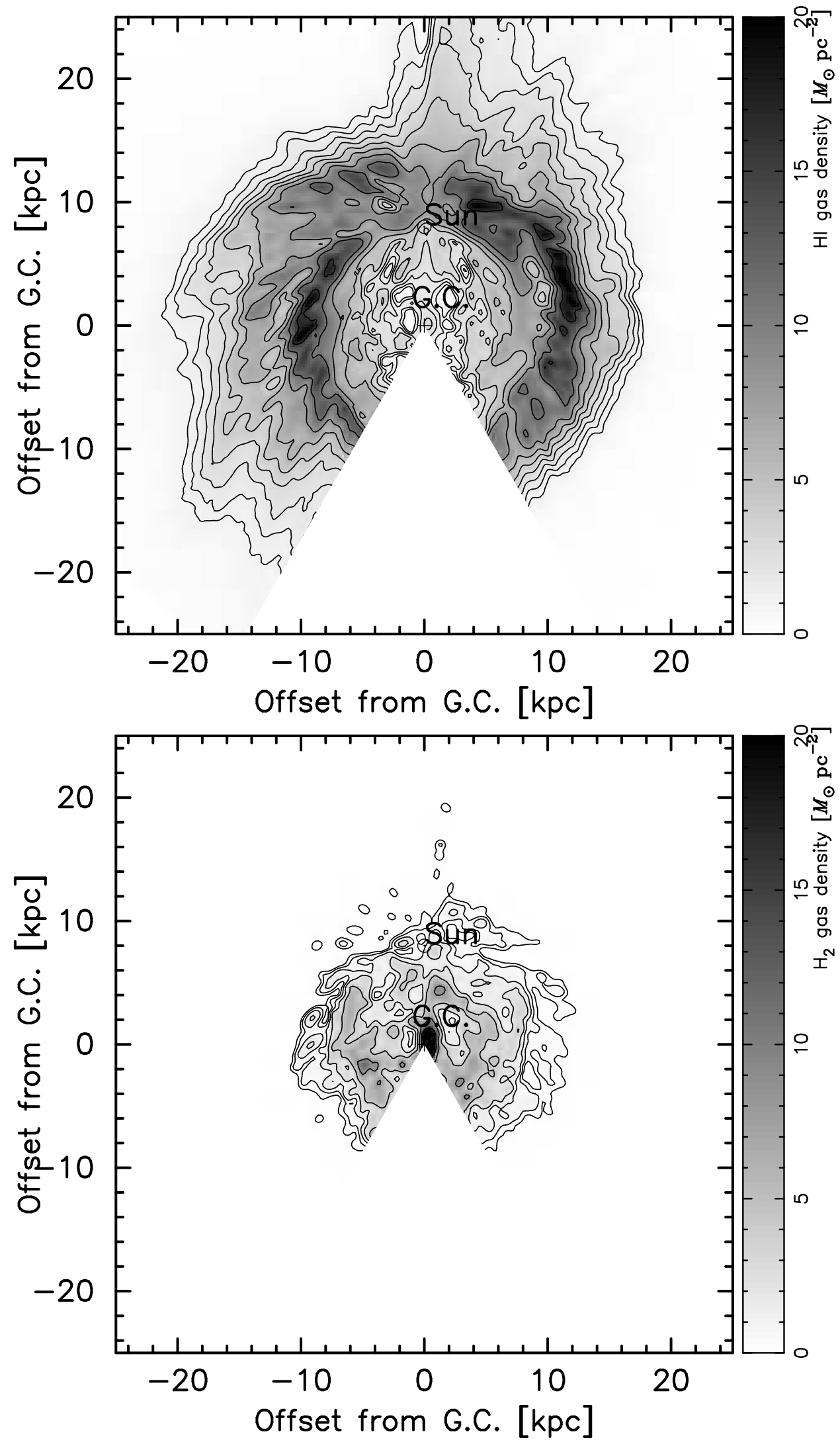

Fig. 1. Face-on maps of (top) $\mathrm{HI}$ and (bottom) $\mathrm{H}_{2}$ gases. Contour levels are 1.0, 1.4, 2.0, 2.8, 4.0, 5.6, 8.0, $11.3 \mathrm{M}_{\odot} \mathrm{pc}^{-2}$ for $\mathrm{HI}$ map and 0.25, 0.5, 1.0, 2.0, 4.0, 8.0, $16.0 \mathrm{M}_{\odot} \mathrm{pc}^{-2}$ for $\mathrm{H}_{2}$. The area of $|\theta|<30^{\circ}$ is masked because the number of points, where $\mathrm{HI}$ and $\mathrm{H}_{2}$ density are calculated, is too small to show the gas distribution. 

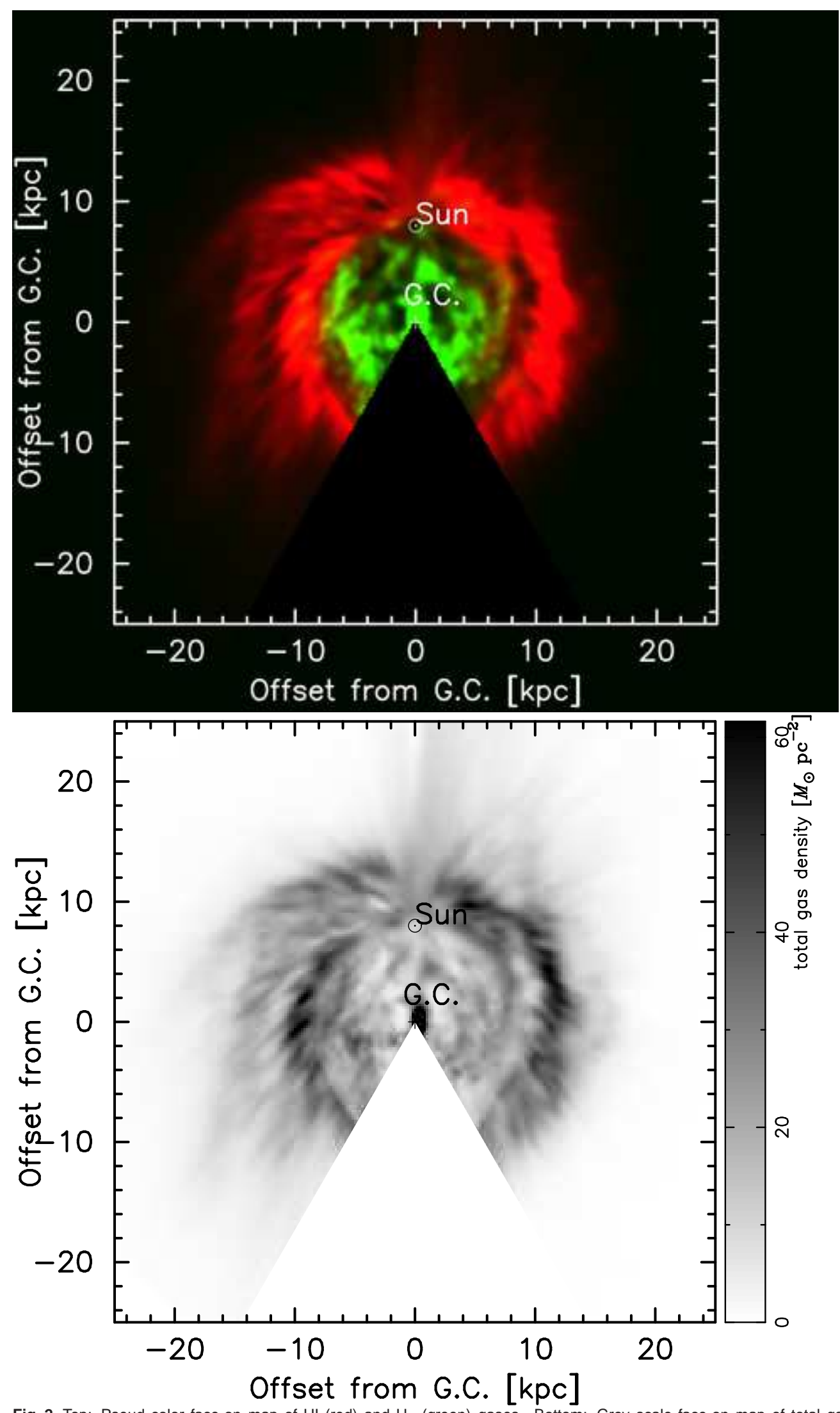

Fig. 2. Top: Pseud color face-on map of $\mathrm{HI}$ (red) and $\mathrm{H}_{2}$ (green) gases. Bottom: Grey scale face-on map of total gas distribution combining $\mathrm{HI}$ and $\mathrm{H}_{2}$ densities. 


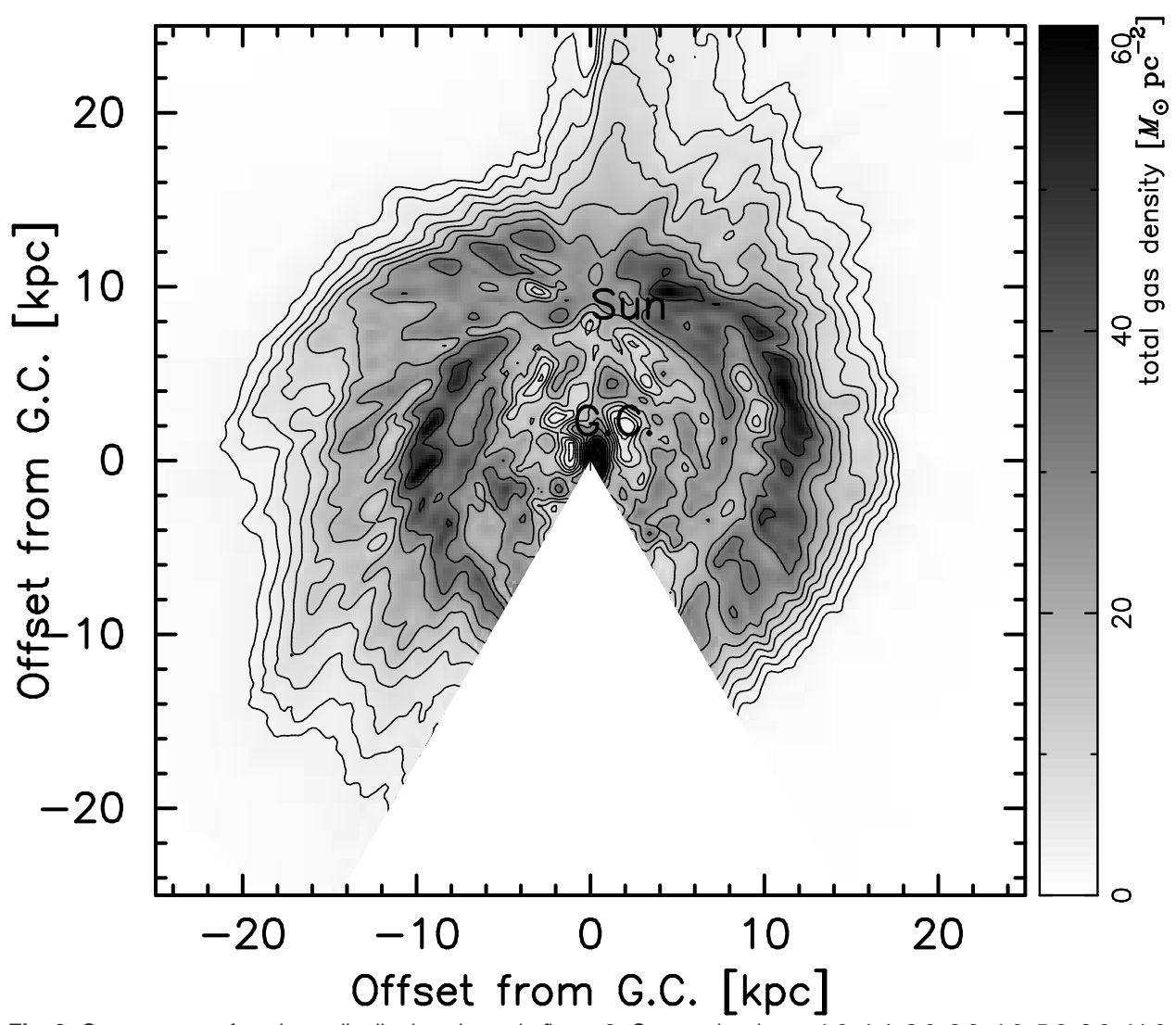

Fig. 3. Contour map of total gas distribution shown in figure 2. Contour levels are 1.0, 1.4, 2.0, 2.8, 4.0, 5.6, 8.0, 11.3, $16.0 \mathrm{M}_{\odot} \mathrm{pc}^{-2}$.

within the solar circle are alomost the same amount of $7.5 \times 10^{8}$ $\mathrm{M}_{\odot}$. This result is similar to Bloemen et al. (1986), who estimated $\mathrm{HI}$ and $\mathrm{H}_{2}$ masses to be $9.2 \times 10^{8} \mathrm{M}_{\odot}$ and $9.4 \times 10^{8}$ $\mathrm{M}_{\odot}$, respectively, by comparing high-energy gamma ray emission. Considering the difference of adopted Galactic constant, their averaged value $9.3 \times 10^{8} \mathrm{M}_{\odot}$ is $9.3 / 7.5 \times(8 / 10)^{2}=0.8$ times smaller than our estimation. Kalberla \& Dedes (2008) estimated the HI mass within the solar circle to be $1.8 \times 10^{9}$ $\mathrm{M}_{\odot}$. This discrepancy is thought to be caused by the difference of adopted models to derive HI distribution in the $z$ direction within the solar circle. In our paper, we took a model with $\operatorname{sech}^{2}$ function as described in the section 3.3. On the other hand, Kalberla \& Dedes (2008) took a model with an exponential function adopted in Kalberla et al. (2007), which can reproduce HI distribution at high altitude. The HI surface density and $\mathrm{HI}$ mass, which we calculated, are thought to be underestimated by the amount of flared HI component as pointed out by Lockman (1984).

$\mathrm{HI}$ gas reaches the maximum value of $7.2 \times 10^{9} \mathrm{M}_{\odot}$ at the radius of $30 \mathrm{kpc}$, which is consistent with Kalberla \& Kerp (2009) considering the difference of adopted Galactic constant $R_{0} . \mathrm{H}_{2}$ reaches the maximum value of $8.5 \times 10^{8} \mathrm{M}_{\odot}$ around the radius of $12 \mathrm{kpc}$. The total gas mass reaches $8.0 \times 10^{9} \mathrm{M}_{\odot}$ at the radius of $30 \mathrm{kpc}$. $\mathrm{HI}$ and $\mathrm{H}_{2}$ gas components are $89 \%$ and $11 \%$ of the total gas mass, respectively.

\subsection{Vertical Cross Sections}

Figure 5 shows vertical cross sections of the $\mathrm{HI}$ and $\mathrm{H}_{2}$ gas distributions. A gray scale and black contours denote the HI density. Red contours represent the $\mathrm{H}_{2}$ gas density.

The $\mathrm{H}_{2}$ gas disk is thinner than the $\mathrm{HI}$ disk as external galaxies shows the same characteristics (Scoville et al. 1993). The thickness of the HI gas disk increases with the Galactocentric distance as former works presented (Levine et al. 2006; Kalberla et al. 2007), while the thickness of the $\mathrm{H}_{2}$ gas disk shows a smaller variation (Bronfman et al. 1988; Nakanishi \& Sofue 2006).

Figure 5 shows that the peaks of the $\mathrm{H}_{2}$ gas distribution roughly coincide with the HI gas peaks. However, midplane of $\mathrm{H}_{2}$ disk is slightly shifted from the $\mathrm{HI}$ midplane in some parts as clearly seen in the outer disk of $\theta=120^{\circ}-140^{\circ}$. This can be interpreted that molecular gas fraction is not determined only with the pressure but UV radiation from star-forming regions changing the molecular fraction (Tanaka et al. 2014) or that it is transient phase, in which molecular fraction leads to a equilibrium (Nakanishi et al. 2006; Inutsuka et al. 2015).

\subsection{Thickness of the Gas Disk}

The thickness of the gas layer is measured for each case of HI and $\mathrm{H}_{2}$ as shown in figure 6. The thickness of HI gas layer 

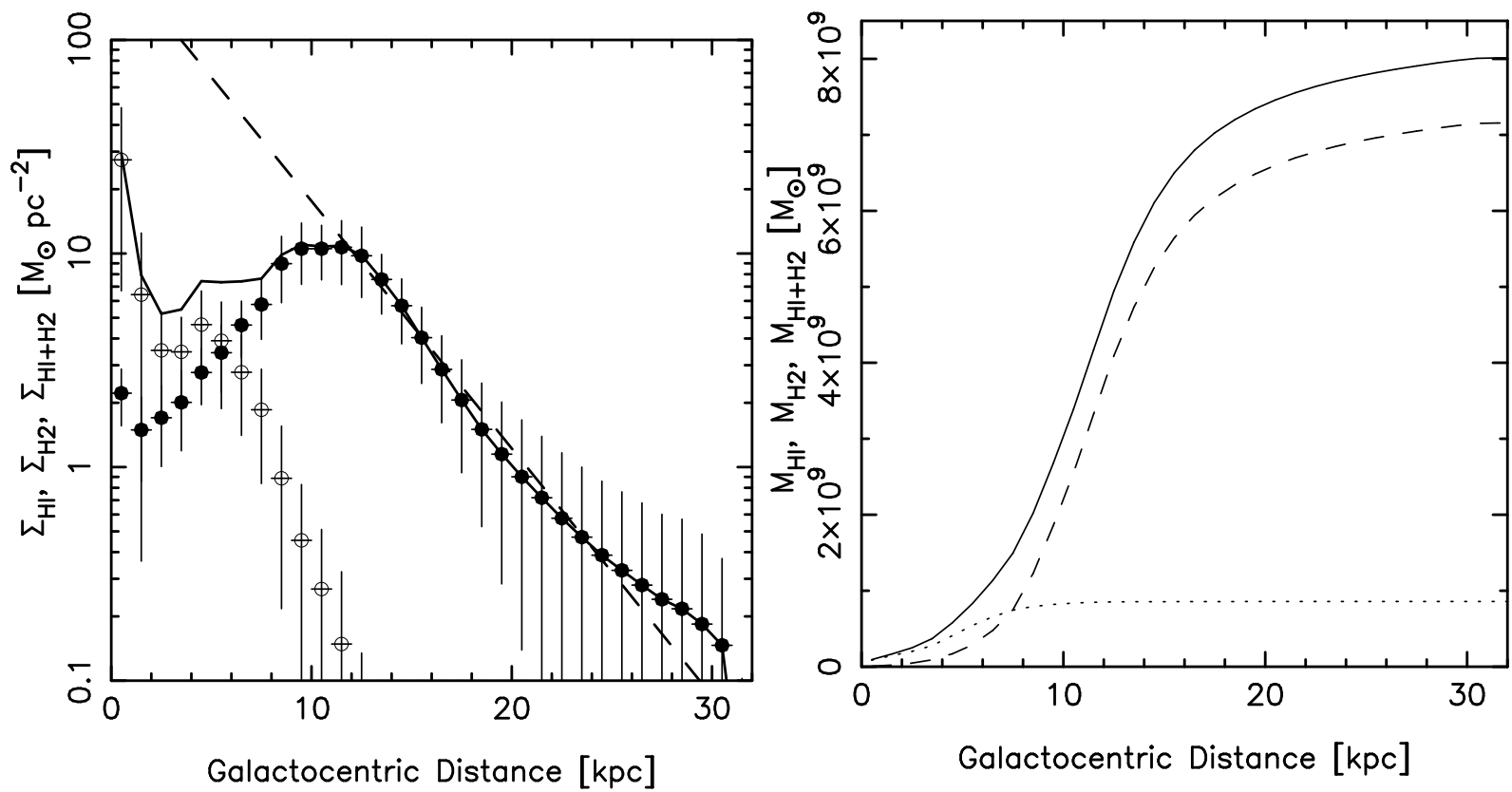

Fig. 4. Left: Radial profiles of $\mathrm{HI}$ (filled circles), $\mathrm{H}_{2}$ (open circles) and total gas surface densities (thick line). Dashed line is the fitted line taken from Kalberla \& Kerp (2009). Right: A function of the accumulated gas mass within each radius. The dashed, dotted, and solid lines denote $\mathrm{HI}, \mathrm{H}_{2}$, and total gases, respectively. $\mathrm{H}_{2}$ and $\mathrm{HI}$ components reach the maximum value at the radii of 10 and $30 \mathrm{kpc}$, respectively.

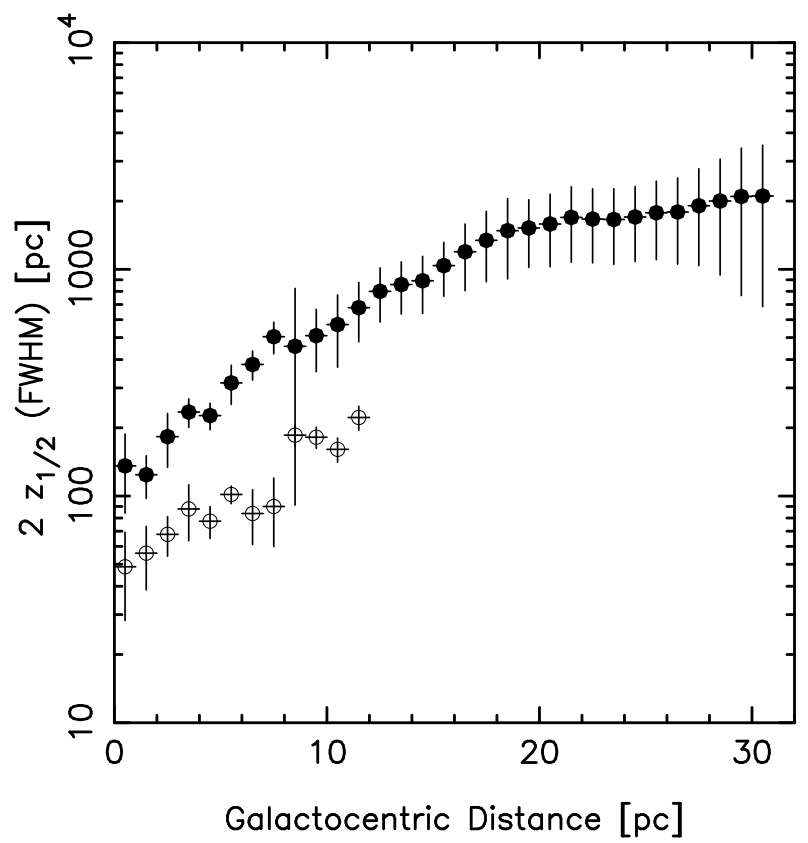

Fig. 6. Thickness of the $\mathrm{HI}$ (filled circle) and $\mathrm{H}_{2}$ (open circle) gas layers measured with $2 z_{1 / 2}$ (FWHM) versus the Galactocentric distance.

increases from $100 \mathrm{pc}$ to $2 \mathrm{kpc}$ with the radius, while the thickness of $\mathrm{H}_{2}$ gas layer slowly increases from $\sim 50 \mathrm{pc}$ to $\sim 200 \mathrm{pc}$. These results are consistent with the previous works (Papers I and II). The thin and small $\mathrm{H}_{2}$ disk is embedded in the thicker and more extended $\mathrm{HI}$ gas disk as shown in a field galaxy (Crosthwaite et al. 2001).

\subsection{Spiral Arms}

The total gas map shown in figure 3 enable us to trace spiral arms from the inner to the outer Galaxy more clearly than ever, by taking advantages of the $\mathrm{HI}$ and $\mathrm{H}_{2}$ maps. We can trace the well-known spiral arms such as the Norma-Cygnus, the Sagittarius-Carina, the Perseus, the Scutum-Crux, and the Orion arms from the inner to the outer Galaxy as shown in figure 7, which shows schematic tracings of all these spiral arms. All the spiral arms can be traced as logarithmic spiral arms, which are overlaid on the total gas map with thick lines. The pitch angles are in the range of $11^{\circ}-15^{\circ}$, which is the consistent with the mean value, $13 .^{\circ} 1 \pm 0.6$, of former works shown by Vallée (2015). The directions of tangents to individual arms are indicated with with broken lines. We describe properties of individual spiral arms in detail below.

\subsubsection{Norma-Cygnus Arm}

The Norma arm is referred as the inner part of the spiral arm labelled with No.1. It is originally identified as a spiral arm found in the fourth quadrant in the inner Galaxy. The tangents to the Norma arm is found in the direction of $l=328.5 \pm 3.5$ (Vallée 2014), which corresponds to the direction of the constellation Norma.

The Cygnus arm is referred as the outer part of the spiral arm labelled with No.1. The Cygnus arm is also called the Outer arm (Vallée 2014). It is clearly traced in the face-on HI map as one of the most prominent arms in the outermost region, which arcs from $(R, \theta) \sim\left(9 \mathrm{kpc}, 50^{\circ}\right)$ to $(R, \theta) \sim\left(11 \mathrm{kpc}, 120^{\circ}\right)$ as 

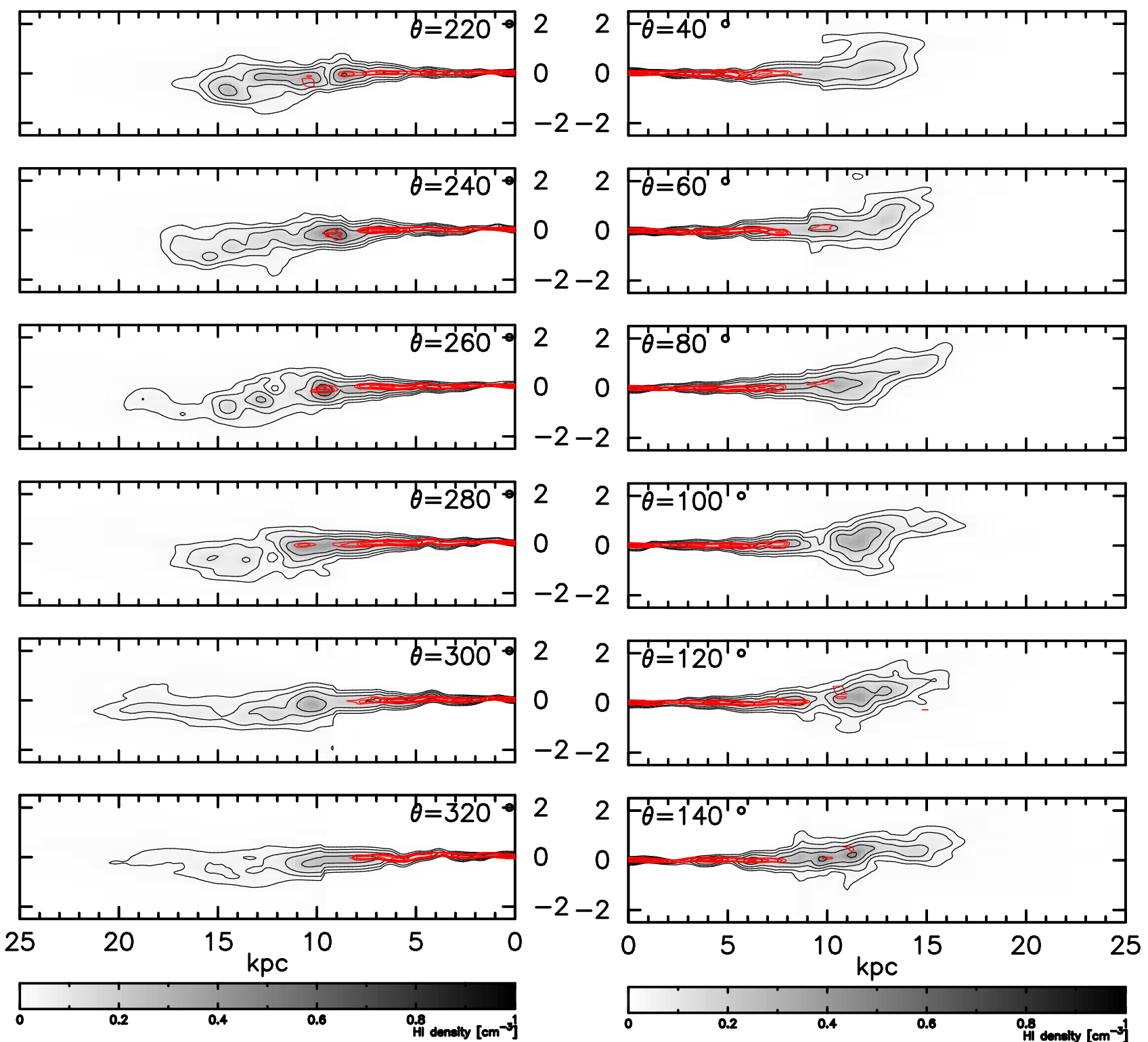

Fig. 5. Vertical slices of $\mathrm{HI}$ and $\mathrm{H}_{2}$ gas maps. $\mathrm{HI}$ distribution is displayed with gray scale and black contours. $\mathrm{H}_{2}$ map is presented with red contours. Contour levels are $0.025,0.05,0.10,0.20$, and $0.40 \mathrm{~cm}^{-3}$.

Table 1. Parameters of Spiral Arms

\begin{tabular}{lcccc}
\hline \hline Name & $\begin{array}{c}\text { Pitch angle } \\
\left({ }^{\circ}\right)\end{array}$ & $\begin{array}{c}\text { Beginning radius } \\
(\mathrm{kpc})\end{array}$ & $\begin{array}{c}\text { Beginning angle } \\
\left({ }^{\circ}\right)\end{array}$ & $\begin{array}{c}\text { Ending angle } \\
\left({ }^{\circ}\right)\end{array}$ \\
\hline Norma-Cygnus (Outer) & 15 & 2.6 & -210 & 130 \\
Perseus & 15 & 2.9 & -120 & 260 \\
Sagittarius-Carina & 11 & 4.2 & 30 & 310 \\
Scutum-Crux & 11 & 2.6 & -30 & 430 \\
Orion (Local) & 15 & 7.0 & 130 & 290 \\
\hline \hline
\end{tabular}




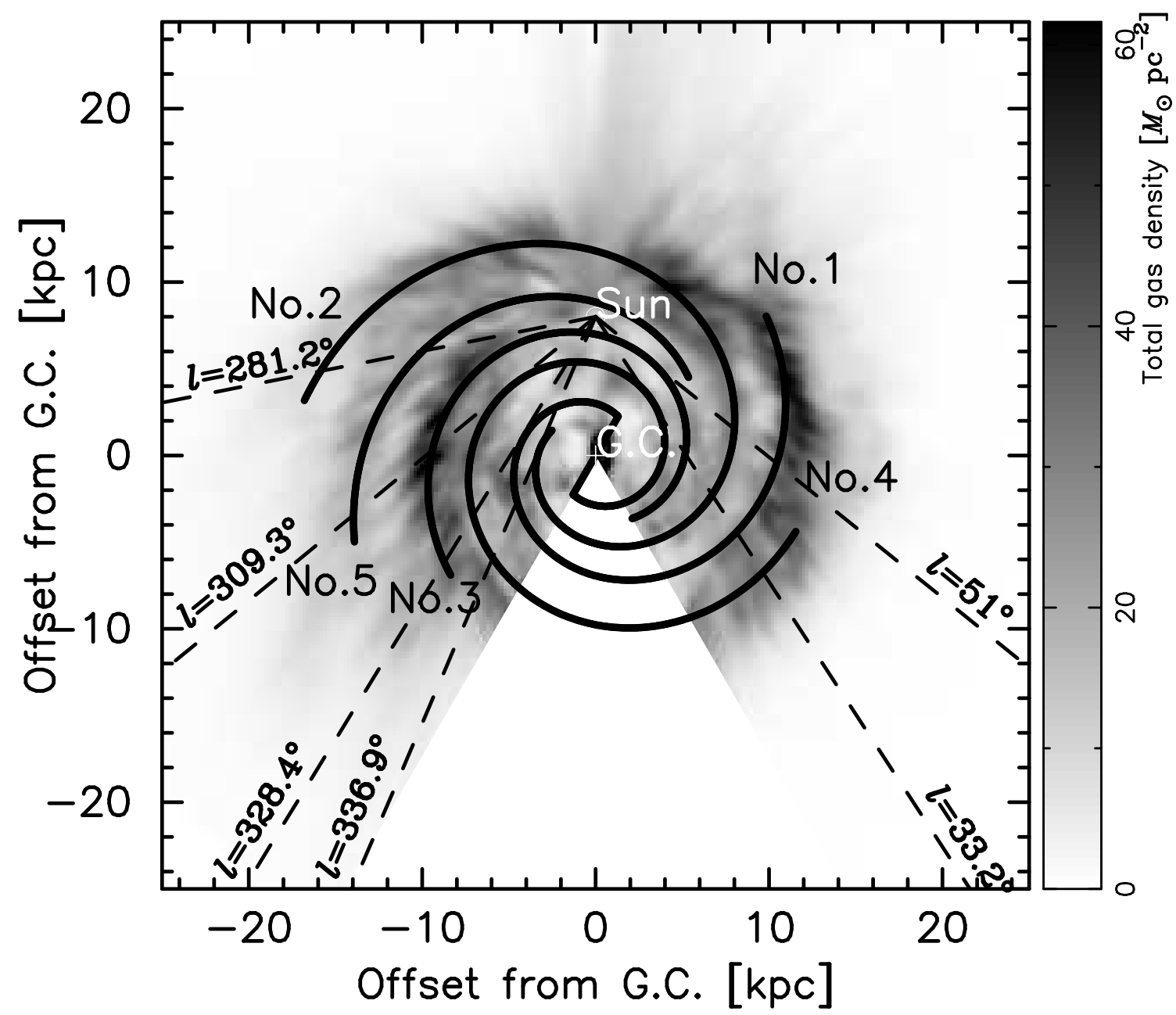

Fig. 7. Schematic tracers of spiral arms superimposed on the total gas distribution map; No.1: Norma-Cygnus arm; No.2: Perseus arm; No.3: SagittariusCarina arm; No.4: Scutum-Crux arm; No.5: Orion (Local) arm. Dashed lines are tangents to the inner spiral arms.

described in Paper II. Recent VLBI observation in $\mathrm{H}_{2} \mathrm{O}$ masers line traced this spiral as a logarithmic spiral arm with pitch angle of $13.8 \pm 3 .{ }^{\circ} 3$ based on the annual parallax (Hachisuka et al. 2015).

As discussed in Paper II, the Norma and the Cygnus arms can be identified as the same spiral arm considering that their pitch angles should be $11^{\circ}-15^{\circ}$. Hence, we call this arm the Norma-Cygnus arm and labeled with No.1 as shown in figure 7 , where a schematic tracing of the Norma-Cygnus arm is presented by a logarithmic spiral arm starting at $(R, \theta)=$ $\left(2.6 \mathrm{kpc},-210^{\circ}\right)$ and ending at $\theta=130^{\circ}$ with a pitch angle of $15^{\circ}$. Its origin is considered to coincide with "near $3 \mathrm{kpc}$ arm" and to connect to the the near side of the Galactic bar (Vallée 2014). The parameters describing the arm is summarizes in Table 1. This picture is consistent with the recent panoramic view obtained with compilation of former works (Vallée 2005).

\subsubsection{Perseus Arm}

The Perseus arm labelled with No. 2 is known as one of the most prominent arms (Georgelin and Georgelin 1976). It is found in the range of $\theta=30^{\circ}-230^{\circ}$, and is prominent in the outer Galaxy. The Perseus arm can be traced with a logarithmic spiral arm with a pitch angle of $15^{\circ}$. In figure 7 , a schematic tracing of the Perseus arm is shown by a logarithmic spiral arm starting at $(R, \theta)=\left(2.9 \mathrm{kpc},-120^{\circ}\right)$ with a pitch angle of $15^{\circ}$ and ending at $\theta=260^{\circ}$.

Vallée (2014) compiled the former works and concluded that the Perseus arm starts at the direction of $l=336 .{ }^{\circ} 9 \pm 0.70 .7$, while Churchwell et al. (2009) concluded that the Perseus arm starts at the far edge of the central bar. If we assume that the Perseus arms start at the far edge of the central bar, the major radius of the central bar needs to be $5 \mathrm{kpc}$, considering the pitch angle and its distance measured around the Sun. However, the size of the bar and expected non-circular motion is inconsistent with former works. Our study supports the picture that the Perseus arm starts at $l=336^{\circ} 9 \pm 0.7$ but not from the either 
edge of the central bar, being concluded by Vallée (2014).

\subsubsection{Sagittarius-Carina Arm}

The Sagittarius-Carina arm labelled with No.3 in figure 7 is known as the closest spiral arm to the Sun. The tangents to the Sagittarius and Carina arms are found in the directions of $l=48.548 .5 \pm 2.5$ and $l=284 .{ }^{\circ} 5 \pm 2 .{ }^{\circ} 5$, which correspond to the directions of the Sagittarius and Carina constellations, respectively (Vallée 2014).

In this study, the side of the Carina arm can be clearly traced in the HI and total gas maps and is well fitted by a logarithmic spiral arm with a pitch angle of $11^{\circ}$, though the side of the Sagittarius arm is less prominent as mentioned in Paper II. In figure 7 a schematic tracing of this arm is presented by a logarithmic spiral arm starting at $(R, \theta)=\left(4.2 \mathrm{kpc}, 30^{\circ}\right)$ with a pitch angle of $11^{\circ}$ and ending at $\theta=310^{\circ}$.

\subsubsection{Scutum-Crux Arm}

The Scutum-Crux arm labelled with No.4 is found between the Norma-Cygnus and the Sagittarius-Carina arms as indicated in figure 7. Similarly to the Sagittarius-Carina arm, the tangent to the Scutum and Crux arms are found in the directions of $l=33.2 \pm 17.2$ and $l=309.3 \pm 0.9$ (Vallée 2014), which correspond to the directions of the Scutum and Crux constellations, respectively. The Crux arm often referred as Centaurus arm and so it is often called Scutum-Centaurus (Churchwell et al. 2009).

In the first quadrant, the separation between the ScutumCrux and Sagittarius-Carina arms gets small and they seem form a ring-like structure, which was reported to exist at the Galactocentric radius of $R_{0} / 2$ (Clemens, Sanders, \& Scoville 1988).

This arm can be traced with a logarithmic spiral arm with a pitch angle of $11^{\circ}$. Considering its pitch angle, it is thought to connect to the distant molecular arm found by Dame \& Thaddeus (2011) as they concluded. In figure 7 a schematic tracing of the Scutum-Crux arm is shown by a logarithmic spiral arm starting at $(R, \theta)=\left(2.6 \mathrm{kpc},-30^{\circ}\right)$ and ending at $\theta=430^{\circ}$ with a pitch angle of $11^{\circ}$.

\subsubsection{Orion Arm (Local Arm)}

Between the Sagittarius-Carina and the Perseus arms, there exists a less prominent but clear spiral arm, where the Sun is located, as shown in figure 7. This spiral arm is the referred as the Orion arm or the Local arm (Bobylev \& Bajkova 2014). In figure 7, a schematic tracing of the Local arm is presented by a logarithmic spiral arm starting at $(R, \theta)=\left(7.0 \mathrm{kpc}, 130^{\circ}\right)$ and ending at $\theta=290^{\circ}$ with a pitch angle of $15^{\circ}$.

So far, there are two possibilities on the structure of the Orion arm; (1) it is a bridge-like structure connecting the Sagittarius-Carina and Perseus arms (Weaver 1970; Churchwell et al. 2009), or (2) normal spiral arm with similar pitch angle as the others. Our result supports the latter picture, which is is consistent with recent VLBI result concluded by Xu et al. (2013) and Bobylev \& Bajkova (2014), who measured annual parallaxes of 23-25 high-mass star formation regions with VLBA to show that the Orion arm is traced with a logarithmic spiral arm with the pitch angle of $12.9 \pm 2.9-13.8 \pm 3.8$.

\subsection{Molecular Fraction}

We here define a parameter $f_{\mathrm{mol}}$ to quantitatively investigate the physical properties of the ISM as follows,

$f_{\mathrm{mol}}=\frac{\Sigma_{\mathrm{H}_{2}}}{\Sigma_{\mathrm{HI}}+\Sigma_{\mathrm{H}_{2}}}$,

where $\Sigma_{\mathrm{HI}}$ and $\Sigma_{\mathrm{H}_{2}}$ are surface densities of $\mathrm{HI}$ and $\mathrm{H}_{2}$ gases, respectively. Figure 8 shows $f_{\text {mol }}$ plotted versus Galactocentric distance. The molecular fraction $f_{\mathrm{mol}}$ is above 0.5 in the Galactocentric range of $(R<5 \mathrm{kpc})$, which implies that $\mathrm{H}_{2}$ gas is dominant in this range. The molecular fraction $f_{\text {mol }}$ rapidly decreses beyond $R=5 \mathrm{kpc}$ and gets less than 0.1 in the outer Galaxy $(R>8 \mathrm{kpc})$, which implies that $\mathrm{HI}$ gas is dominant in the outer disk.

Figure 9 shows a map of two-dimensional $f_{\mathrm{mol}}$ distribution. In figure 9 we superimposed schematic tracings of spiral arms with gray lines. These spiral arms are the same as those described in Section 4.1. In addition to the global variation, local variations of the molecular fraction are found. The molecular fraction $f_{\text {mol }}$ tends to increase along the spiral arms rather than the inter-arm regions. The molecular fraction $f_{\text {mol }}$ varies by 0.1 -0.2 between an arm and an inter-arm. This tendency is also found in external galaxies Kuno et al. (1995).

\section{Possible Uncertainties}

We adopted several assumptions for the simplicity to obtain the gaseous maps. In this section, we note possible systematic errors caused by such assumptions.

\section{Kinematic Distance}

The kinematic distance, which we adopted as a distance estimator, is based on the assumption that the Galactic rotation is purely circular. However, it is known that non-circular motion exists and affects the kinematic distance.

There have been a lot of efforts to the measurements of annual parallaxes with VLBI, which have shown that typical error is within a few kpc (Hachisuka et al. 2009; Sakai et al. 2012; Nakanishi et al. 2015). Figure 10 shows comparison of VLBI trigonometric distance taken from Reid et al. (2014) and kinematic distance used in this paper. Open and filled circles indicate sources within the Galactocenric distance of $3 \mathrm{kpc}$ and the others, respectively. If the both values are the same, the data are plotted on the linear line. 


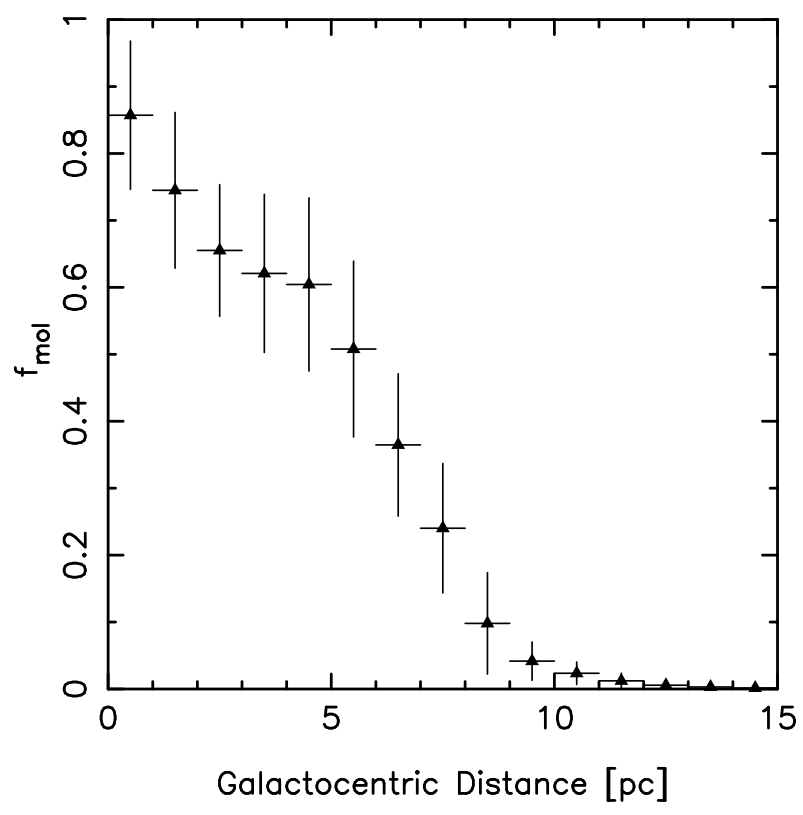

Fig. 8. Radial variation of the molecular fraction $\left(f_{\text {mol }}\right)$ obtained by azimuthally averaging two dimensional $f_{\mathrm{mol}}$ map.

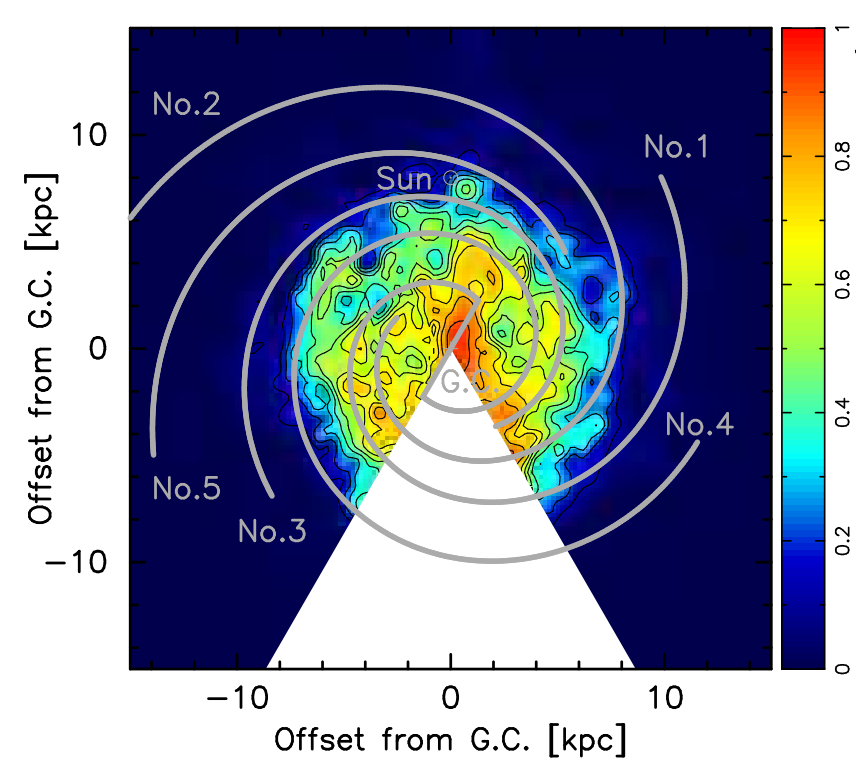

Fig. 9. Two-dimensional molecular fraction $\left(f_{\mathrm{mol}}\right)$ map calculated with $\mathrm{HI}$ and $\mathrm{H}_{2}$ surface densities. The molecular faction $f_{\mathrm{mol}}$ is defined as $f_{\mathrm{mol}}=$ $\Sigma_{\mathrm{H}_{2}} /\left(\Sigma_{\mathrm{HI}}+\Sigma_{\mathrm{H}_{2}}\right)$. The schematic tracers of spiral arms are indicated with gray lines.
The standard deviation between trigonometric and kinemactic distances is calculated to be $2.3 \mathrm{kpc}$, which is a typical error in heliocentric distance of the maps shown in this paper. This value is consistent with results from the recent an $\mathrm{N}$ body+hydrodynamical simulation shown by Baba et al. (2009), who concluded that kinematic distance had error of 2-3 kpc.

Figure 10 shows that there is no big difference between filled and open circles though the non-circular motion is significant near the Galactic center (e.g., Sawada et al. 2004). Therefore, the typical error of the kinemetic distance of the sources within the Galactocentric distance of $3 \mathrm{kpc}$ is of the same order of the other sources located in the outer region.

\section{Galactic Constant}

The Galactic constants $\left(R_{0}, V_{0}\right)$ change the kinematic distance, according to the equation (1). Since the Galactocentric distance of a gas cloud having LSR velocity $V_{\mathrm{LSR}}$ in the direction of galactic longitude $l$ is calculated to be $R=R_{0} /\left(\frac{V_{\mathrm{LSR}}}{V(R) \sin l}+\right.$ $\left.\frac{V_{0}}{V(R)}\right)$, the solar Galactocentric distance $R_{0}$ essentially scales the size but not the shape of the gaseous disk. The surface density changes by square of $R_{0}$.

Though the solar rotational velocity $V_{0}$ can change the kinematic distance, it is order of $V_{0} / V(R)$, which is typically less than $25 \%$ as is estimated from fluctuations of the rotation curve (Sofue et al. 2009).

\section{Fitting Method}

In order to divide the mixture emission from near and far points of the inner Galaxy, we utilized the fitting method as explained in section 3.3. However, it is not necessarily perfect because the actual HI vertical distribution cannot expressed with a single 4 高 function (Lockman 1984). A difference of midplane density between the data and model is typically less than $20-30 \%$ as mentioned in Paper II.

\section{Optical Thickness}

In this study, we assume that the HI line is optically thin to measure the HI column density for the simplicity. However, recent studies point out that $\mathrm{HI}$ mass is underestimated by factor 2-2.5 when HI line is assumed to be optically thin (Fukui et al. 2014; Fukui et al. 2015).

\section{Summary}

In this paper, we showed three dimensional $\mathrm{HI}$ and $\mathrm{H}_{2}$ maps of the Milky Way Galaxy using the latest HI and CO survey data and rotation curves based on the kinematic distance. The HI map has been revised with the improved analysis from the Paper I.

Comparing the $\mathrm{HI}$ and $\mathrm{H}_{2}$ maps, it was shown that the $\mathrm{H}_{2}$ gas is distributed mainly inside the solar circle while HI distribution is extended to the large Galactocentric distance of 15$20 \mathrm{kpc}$ and its outskirt is asymmetric about the Galactic center. The radial distribution of total gas density generally decreases 


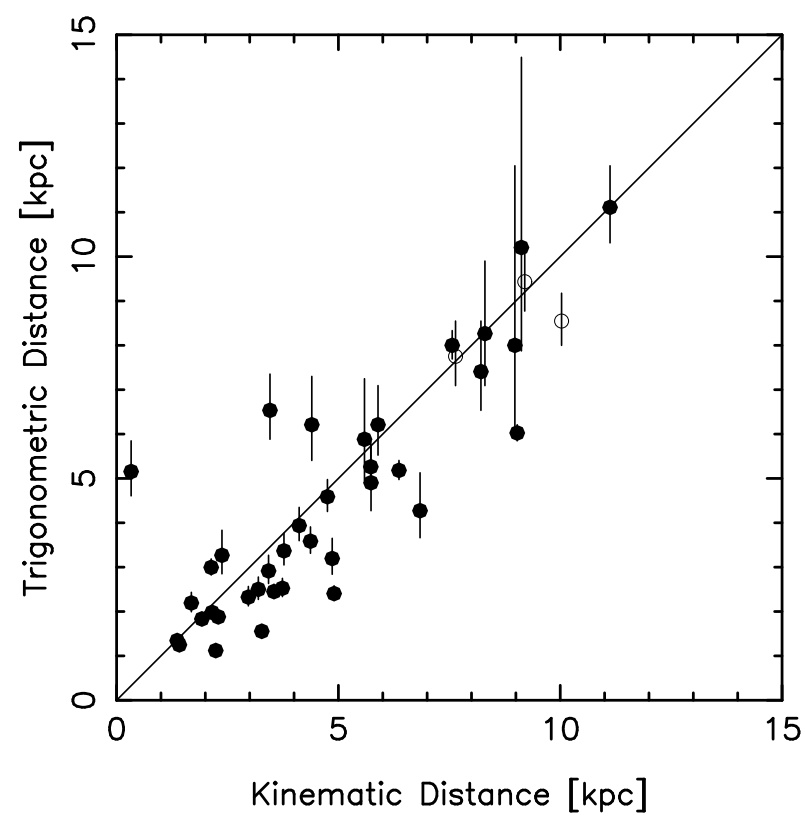

Fig. 10. Comparison of kinematic distance used in this paper and trigonometric distance obtained with VLBI observation (Reid et al. 2014). Open and filled circles indicate sources within the Galactocenric distance of $3 \mathrm{kpc}$ and the others, respectively.

with the Galactocentric distance peaking at the center while it is roughly constant between 5 and $10 \mathrm{kpc}$. The total gas mass is $8 \times 10^{9} \mathrm{M}_{\odot}, 89 \%$ and $11 \%$ of which are $\mathrm{HI}$ and $\mathrm{H}_{2}$ gas masses, respectively. The $\mathrm{H}_{2}$ gas layer is embedded in the thicker and more extended HI layer. The HI layer is largely bent at the Galactocentric distance of $12 \mathrm{kpc}$ as well as is corrugated in the inner Galaxy.

We show the total gas density map of the Milky Way Galaxy for the first time by summing up the $\mathrm{HI}$ and $\mathrm{H}_{2}$ gas density map. It is more advantageous to trace spiral arms from the center to the outer Galaxy than the individual $\mathrm{HI}$ and $\mathrm{H}_{2}$ maps. We could easily trace five logarithmic spiral arms in the total gas map such as the Norma-Cygnus, the Perseus, the SagittariusCarina, the Scutum-Crux, and the Orion arms. The Norma and the Cygnus arms are identified as the same spiral arm, considering their pitch angles of $11^{\circ}-15^{\circ}$.

The fraction of molecular component to the total gas decreases with the Galactocentric distance and drops steeply in the small radius range of $5-8 \mathrm{kpc}$. The two dimensional molecular fraction map shows that it is also locally enhanced on the spiral arms.

Acknowledgement We would like to thank the referee for carefully reading the manuscript. This work was supported by JSPS KAKENHI Grant Number 26800104.

\section{References}

Arimoto, N., Sofue, Y., and Tsujimoto,T. 1996, PASJ, 48, 275
Baba, J., Asaki, Y., Makino, J., et al. 2009, ApJ, 706, 471

Bajaja, E., Arnal, E. M., Larrarte, J. J., et al. 2005, A\&A, 440, 767

Beuermann, K., Kanbach, G., \& Berkhuijsen, E. M. 1985, A\&A, 153, 17

Bitran, M., Alvarez, H., Bronfman, L., May, J., \& Thaddeus, P. 1997, A\&AS, 125, 99

Bloemen, J. B. G. M., Strong, A. W., Mayer-Hasselwander, H. A., et al. 1986, A\&A, 154, 25

Bobylev, V. V., \& Bajkova, A. T. 2014, Astronomy Letters, 40, 783

Broeils, A. H., \& van Woerden, H. 1994, A\&AS, 107, 129

Bronfman, L., Cohen, R. S., Alvarez, H., May, J., \& Thaddeus, P. 1988, ApJ, 324, 248

Bronfman, L., Alvarez, H., Cohen, R. S., \& Thaddeus, P. 1989, ApJS, 71, 481

Burton, W. B., \& Liszt, H. S. 1983, A\&AS, 52, 63

Caswell, J. L., Fuller, G. A., Green, J. A., et al. 2011, MNRAS, 417, 1964

Chen, W., Gehrels, N., Diehl, R., \& Hartmann, D. 1996, A\&AS, 120, 315

Churchwell, E., Babler, B. L., Meade, M. R., et al. 2009, PASP, 121, 213

Clemens, D. P. 1985, ApJ, 295, 422

Clemens, D. P., Sanders, D. B., \& Scoville, N. Z. 1988, ApJ, 327, 139

Crosthwaite, L. P., Turner, J. L., Hurt, R. L., et al. 2001, AJ, 122, 797

Cohen, R. S., Grabelsky, D. A., May, J., et al. 1985, ApJL, 290, L15

Dame, T. M., Elmegreen, B. G., Cohen, R. S., \& Thaddeus, P. 1986, ApJ, 305,892

Dame, T. M. et al. 1987, ApJ, 322, 706

Dame, T. M., Hartmann, D., \& Thaddeus, P. 2001, ApJ, 547, 792

Dame, T. M., \& Thaddeus, P. 2008, ApJL, 683, L143

Dame, T. M., \& Thaddeus, P. 2011, ApJL, 734, L24

Dehnen, W. and Binney, J. 1998, MNRAS, 294, 429

Downes, D., Wilson, T. L., Bieging, J., \& Wink, J. 1980, A\&AS, 40, 379

Drimmel, R., Spergel, D. N. 2001, ApJ, 556, 181

Fukui, Y., Okamoto, R., Kaji, R., et al. 2014, ApJ, 796, 59

Fukui, Y., Torii, K., Onishi, T., et al. 2015, ApJ, 798, 6

Georgelin, Y. M., and Georgelin, Y. P. 1976, A\&A, 49, 57

Grabelsky, D. A., Cohen, R. S., Bronfman, L., Thaddeus, P., \& May, J. 1987, ApJ, 315, 122

Hachisuka, K., Brunthaler, A., Menten, K. M., et al. 2009, ApJ, 696, 1981

Hachisuka, K., Choi, Y. K., Reid, M. J., et al. 2015, ApJ, 800, 2

Hartmann, D., \& Burton, W. B. 1997, Atlas of Galactic Neutral Hydrogen, by Dap Hartmann and W. Butler Burton, pp. 243. ISBN 0521471117. Cambridge, UK: Cambridge University Press, February 1997.,

Heiles, C. 1979, ApJ, 229, 533

Honma, M., Sofue, Y., \& Arimoto, N. 1995, A\&A, 304, 1

Imamura, K., \& Sofue, Y. 1997, A\&A, 319, 1

Inutsuka, S.-i., Inoue, T., Iwasaki, K., \& Hosokawa, T. 2015, arXiv: 1505.04696

Kalberla, P. M. W., Burton, W. B., Hartmann, D., et al. 2005, A\&A, 440, 775

Kalberla, P. M. W., Dedes, L., Kerp, J., \& Haud, U. 2007, A\&A, 469, 511

Kalberla, P. M. W., \& Dedes, L. 2008, A\&A, 487, 951

Kalberla, P. M. W., \& Kerp, J. 2009, ARA\&A, 47, 27

Kerr, F. J. 1969, ARA\&A, 7, 39

Kerr, F. J., Bowers, P. F., Jackson, P. D., \& Kerr, M. 1986, A\&AS, 66, 373

Kuno, N., Nakai, N., Handa, T., \& Sofue, Y. 1995, PASJ, 47, 745

Levine, E. S., Blitz, L., \& Heiles, C. 2006, ApJ, 643, 881

Lockman, F. J. 1984, ApJ, 283, 90

May, J., Bronfman, L., Alvarez, H., Murphy, D. C., \& Thaddeus, P. 1993, A\&AS, 99, 105 
McClure-Griffiths, N. M., Dickey, J. M., Gaensler, B. M., \& Green, A. J. 2004, ApJL, 607, L127

Myers, P. C., Dame, T. M., Thaddeus, P., Cohen, R. S., Silverberg, R. F., Dwek, E., \& Hauser, M. G. 1986, ApJ, 301, 398

Muller, C. A., \& Westerhout, G. 1957, Bull. Astron. Inst. Netherlands, 13, 151

Myers, P. C. 1978, ApJ, 225, 380

Nakanishi, H. \& Sofue, Y. 2003, PASJ, 55, 191

Nakanishi, H., \& Sofue, Y. 2006, PASJ, 58, 847

Nakanishi, H., Kuno, N., Sofue, Y., et al. 2006, ApJ, 651, 804

Nakanishi, H., Sakai, N., Kurayama, T., et al. 2015, PASJ, 176

Oort, J. H., Kerr, F. J., \& Westerhout, G. 1958, MNRAS, 118, 379

Reid, M. J., Menten, K. M., Brunthaler, A., et al. 2014, ApJ, 783, 130

Sakai, N., Honma, M., Nakanishi, H., et al. 2012, PASJ, 64, 108

Sanders, D. B., Solomon, P. M., \& Scoville, N. Z. ApJ, 276, 182

Sanders, D. B., Clemens, D. P., Scoville, N. Z., \& Solomon, P. M. 1986, ApJS, 60, 1

Sawada, T., Hasegawa, T., Handa, T., \& Cohen, R. J. 2004, MNRAS, 349, 1167

Scoville, N. Z., Thakkar, D., Carlstrom, J. E., \& Sargent, A. I. 1993, ApJL, 404, L59

Sofue, Y., Honma, M., \& Arimoto, N. 1995, A\&A, 296, 33

Sofue, Y., Honma, M., \& Omodaka, T. 2009, PASJ, 61, 227

Spitzer, L. J. 1942, ApJ, 95, 329

Tanaka, A., Nakanishi, H., Kuno, N., \& Hirota, A. 2014, PASJ, 66, 66

Taylor, J. H., \& Cordes, J. M. 1993, ApJ, 411, 674

Vallée, J. P. 2005, AJ, 130, 569

Vallée, J. P. 2014, ApJS, 215, 1

Vallée, J. P. 2015, MNRAS, 450, 4277

Verschuur, G. L. 1973, A\&A, 22, 139

Wakker, B. P., \& van Woerden, H. 1997, ARA\&A, 35, 217

Weaver, W. B. 1970, AJ, 75, 938

Xu, Y., Li, J. J., Reid, M. J., et al. 2013, ApJ, 769, 15 